\title{
A Comparison of Impact of Chronic Periodontal Diseases and Nonsurgical Periodontal Therapy on Oral Health-Related Quality of Life
}

\author{
Khushboo Goel ${ }^{1}$ and Dharnidhar Baral ${ }^{2}$ \\ ${ }^{1}$ Department of Periodontology and Oral Implantology, B.P. Koirala Institute of Health Sciences, College of Dental Surgery, \\ Dharan 56700, Nepal \\ ${ }^{2}$ School of Public Health and Community Medicine, B.P. Koirala Institute of Health Sciences, Dharan 56700, Nepal
}

Correspondence should be addressed to Khushboo Goel; khushboo.goel@bpkihs.edu

Received 21 November 2016; Revised 10 April 2017; Accepted 20 April 2017; Published 14 May 2017

Academic Editor: Manuel Lagravere

Copyright (C) 2017 Khushboo Goel and Dharnidhar Baral. This is an open access article distributed under the Creative Commons Attribution License, which permits unrestricted use, distribution, and reproduction in any medium, provided the original work is properly cited.

\begin{abstract}
Objectives. To evaluate the impact of chronic periodontal diseases (PDs) and compare phases of nonsurgical periodontal therapy (NSPT) on oral health-related quality of life (OHRQoL) in patients attending a tertiary care center of eastern Nepal. Materials and Methods. Matched for socioeconomic status, participants were recruited in two groups: moderate-to-severe chronic periodontitis ( $n=24,43 \pm 46$ years) and chronic gingivitis $(n=25,30 \pm 96$ years). The treatment modalities were scaling and root surface debridement (RSD) and supragingival scaling, respectively. The impact of periodontal disease treatment status was assessed by a self-reported questionnaire of Nepali Oral Health Impact Profile (OHIP-14) at baseline and 9-12 weeks after NSPT. Results. The median (IQR) OHIP-14 total scores for PDs reduced from 7 (3-11) to 3 (1-7.5) after NSPT. Both groups showed a significant improvement on OHRQoL ( $p$ value $<0.001$ ). The periodontitis group showed an increased median (IQR) reduction of $52 \%$ (35.22-86.15) compared with the gingivitis group with $27 \%(0.00-50.00)$. The impact on orofacial pain, orofacial appearance, and psychosocial dimensions was observed, which improved after NSPT in both groups. Conclusion. PDs are directly associated with OHRQoL and treatment of the disease may enhance quality of life from a patient's perspective. Scaling and RSD provided better influence on OHRQoL than supragingival scaling.
\end{abstract}

\section{Introduction}

There have been dramatic improvements in oral health states in recent decades, but periodontal disease has remained prevalent and with little signs of improvement in the severity of the disease [1]. Periodontal diseases (PDs) cause tooth loss at the end stage of the disease but, ironically at an early stage, no to very few symptoms (swelling, bleeding, and pain) are reported by patients. Therefore, PDs are considered a "silent form of disease" that further impedes tooth preservation [2]. However, recently, their occurrence has been shown to have a substantial sociobehavioral component and, thus, they are considered not only to be a threat to the dentition, but also to affect oral health-related quality of life (OHRQoL) [3]. OHRQoL is defined as a multidimensional build that reflects people's comfort when eating, sleeping, and engaging in social dealings; their self-esteem; and contentment with respect to their oral health [4].

Patient based outcomes are becoming increasingly popular as their opinions differ from true clinical end points of gain in clinical attachment level and decrease in probing pocket depth $[5,6]$. Symptoms such as bleeding gums, tooth mobility, drifting teeth, and unaesthetic loss of anterior papilla can be a symbolic oral health-related problem as it can compromise the ability of the periodontal disease person to eat, speak, socialize, and do various daily activities [5]. These patient perspectives can act as significant indicators to be assessed and help identify individual needs to achieve a holistic approach towards oral health care.

The extent of the impacts could be assessed by different OHRQoL measures [7-9]. Oral Health Impact Profile (OHIP-14) is one of the well validated measures of 
OHQoL [10]. It is considered good at predicting broader psychological well-being and life satisfaction and its sensitivity to change has also been assessed [11]. OHIP-14 can assess the impact of periodontal disease from a patient's perspective and can detect changes in quality of life (QoL) before and after therapy [12]. It is considered to have better internal consistency reliability as it has more items [13] than other measures of OHRQoL [7]. Currently, OHIP14 has been validated for a wide range of populations in different countries and a translated Nepali version of the short form of OHIP-14 questionnaire was used to measure the QoL in the Nepalese adult population [14]. The impact of periodontitis on OHRQoL measures is well recognized worldwide $[6,12,15]$. It is now, however, important to consider its implementation in clinical practice.

Periodontal treatment may be in the form of nonsurgical or surgical therapy. Change in OHRQoL after surgical therapy shows contradictory reports $[16,17]$. Similarly, routine nonsurgical periodontal therapy (NSPT) reports conflicting results of either significant $[6,18]$ or insignificant improvement $[19,20]$. In this part of the world where awareness is limited among people, it is unclear whether periodontal diseases have an influence on their social and psychological well-being. The results are also ambiguous on whether periodontal therapy has a positive effect or whether intervention by a therapist is beneficial for improvement in QoL [20]. Therefore, the aim of this study was to evaluate the impact of NSPT on oral health-related quality of life in periodontal disease patients visiting a tertiary care center in eastern Nepal. Limited data is available over the impact of distinct phases of nonsurgical periodontal treatment [21, 22]; therefore, this study also aims to compare the treatment of nonsurgical periodontal therapy on OHRQoL in gingivitis and periodontitis patients, respectively.

\section{Materials and Methods}

2.1. Study Design. This was a comparative cross-sectional study. This research project was approved by the Institutional Ethics Committee of B.P. Koirala Institute of Health Sciences (BPKIHS), Dharan, Nepal (code number IRC/494/015), in accordance with the ethical principles of the Declaration of Helsinki. This is a tertiary health care center with a tertiary dental hospital as well.

2.2. Study Setting. This study was carried out at the Department of Periodontology and Oral Implantology, College of Dental Surgery, B.P. Koirala Institute of Health Sciences, Dharan, Nepal.

2.3. Criteria for Selection. The Inclusion criteria were as follows: (1) periodontitis group with clinical diagnosis of moderate-to-severe generalized chronic periodontitis, with at least one tooth having pocket depths $(\mathrm{PD}) \geq 5-7 \mathrm{~mm}$ with $\geq 3 \mathrm{~mm}$ attachment loss in different quadrants (either anterior or posterior), or gingivitis group with clinical diagnosis of generalized chronic gingivitis defined as inflammation of the gingiva with no loss of attachment; (2) presence of at least
16 teeth with exclusion of third molars; (3) no extensive periodontal therapy in the previous 6 months; (4) absence of any known systemic illness; (5) wearing a denture or an orthodontic appliance, having caries or other oral or systemic diseases, taking multiple medications, or any adverse habits.

Exclusion criteria were as follows: (1) diagnosed case of aggressive periodontitis, (2) pregnant and lactating females, (3) smoking, and (4) refusal to provide informed consent.

2.4. Sample Size and Sampling Method. The ideal sample size to ensure adequate power for this study was calculated considering the mean for group before and after treatment as 41.08 and 27.68 and standard deviation as 6.80 and 6.93, respectively, with true difference of 1 . Based on the above values and using a formula to estimate the sample size of two means, this study considered a total of fifty participants necessary to provide $80 \%$ power at $95 \%$ confidence interval $(\alpha=0.05)$.

2.5. Methods of Data Collection. One periodontist performed the comprehensive periodontal examination under artificial light with the help of a mouth mirror and periodontal probe (University of North Carolina-15, Hu-Friedy Instruments, Chicago, IL, USA). After baseline examination and necessary tooth extractions, a total of 50 participants were enrolled in this study for both groups. Written consent was obtained from all the participants. Figure 1 displays the study design and the flow of subjects (Figure 1).

Data was collected using an ordered questionnaire which contained information about sociodemographic characteristics. This included age, sex, frequency of brushing, and previous dental visits. Socioeconomic status included information about education, occupation, and family income per month (in Rs) which is a modification of Kuppuswamy's Socioeconomic Status Scale in context to Nepal [23]. There are different approaches to measure OHRQoL; the most popular one is multiple item questionnaires. To measure the impact of periodontal diseases on the quality of life, a psychosocial instrument questionnaire of short form of Nepali version of Oral Health Impact Profile-14 (OHIP-14) item score was used. For usage in Nepalese populations, the OHIP-14 was translated from the original English version and validated in Nepali. It consisted of a set of 14-item scores with high reliability of Cronbach's alpha value of $>0.83$ and validity obtained by doing factorial analysis of the scale. This short form of the original 49-item score [10] provides slightly less information but involves less administration and was found to be as reliable as the original questionnaire [14]. The original publication of the OHIP also categorized items into seven domains and named them as Functional Limitations, Physical Pain, Psychological Discomfort, Physical Disability, Psychological Disability, Social Disability, and Handicap and the 14 questions were two from each of the seven domains $[10,24]$. These domains were conceptually based on the model of oral health and expert opinion rather than on statistical procedures such as the exploratory factor analysis (EFA) [25]; therefore, John et al. in 2014 evidenced a more differentiated four-dimensional structure of OHIP, namely, oral function, orofacial pain, orofacial appearance, and psychosocial 


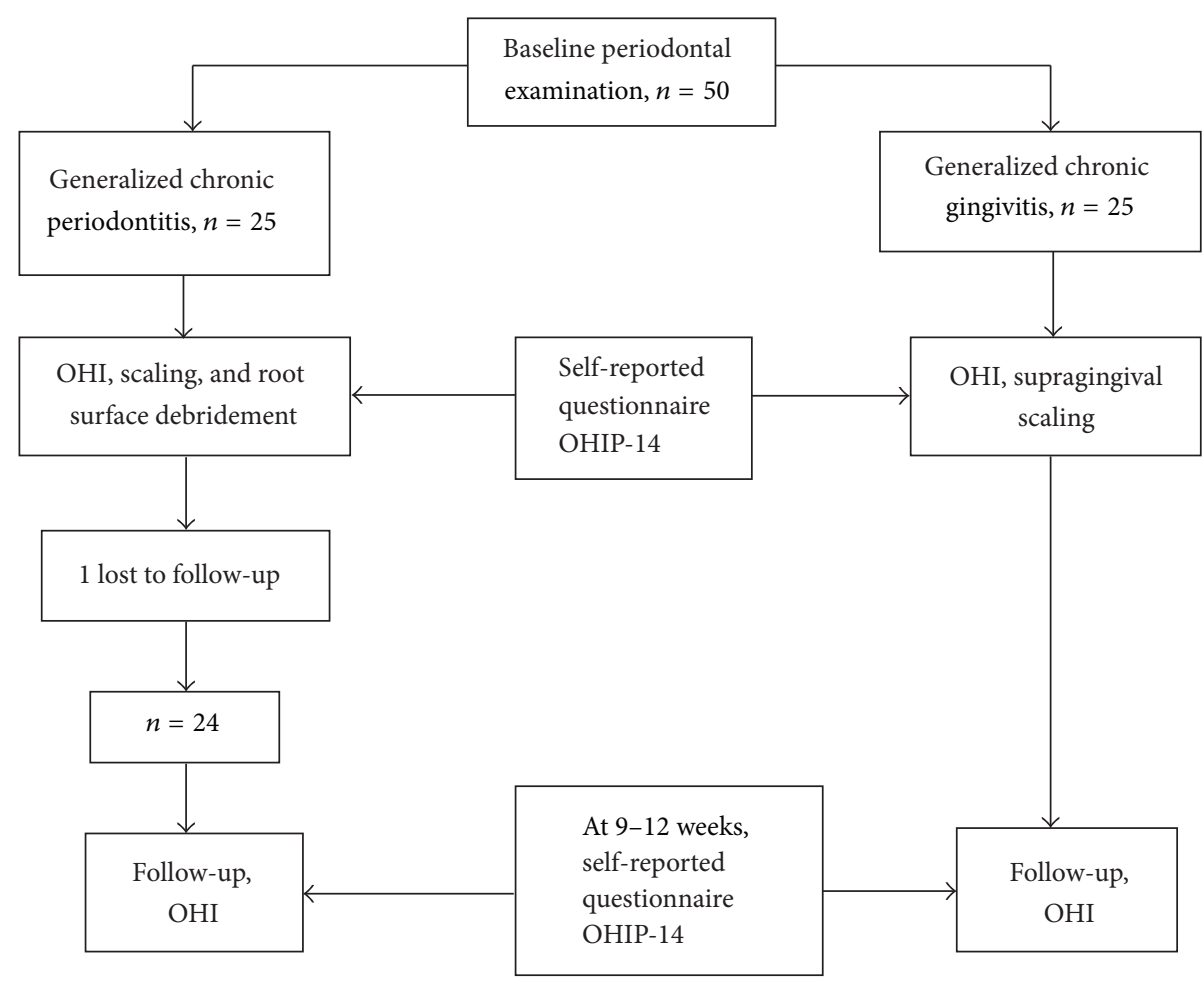

FIGURE 1: Flow chart of study. OHI: oral hygiene instructions; OHIP-14: Oral Health Impact Profile-14.

impact, which is similar across cultures and populations [26]. However, one of the important factors in deciding which of the systems should be used is its validation for the population where the study is to be conducted. Therefore, the Nepali Oral Health Impact Profile (OHIP-14) was used in a questionnaire format and these questions were discussed based on the fourdimensional structure of OHIP. Questions were answered on a Likert scale from 0 to 4 , with $0=$ never, $1=$ hardly ever, $2=$ occasionally, $3=$ fairly often, and $4=$ very often. The summary score will range from "0" to a maximum of " 56 ." " 0 " represents no problems while higher scores represent poor or impaired oral health-related quality of life.

OHIP-14 was self-completed by all participants of both groups at baseline. Both groups received oral hygiene instructions (OHI) at baseline. The periodontitis group received full mouth scaling and root surface debridement (RSD). Supragingival calculus was defined as calcified deposits that are located on the exposed crown and root surfaces and that extend up to $1 \mathrm{~mm}$ below the free gingival margin. An ultrasonic device (Cavitron, Dentsply, York, PA) was utilized to remove supragingival calculus in the first session, and instructions were provided on correct tooth brushing and the use of interproximal brushes. After two weeks, subgingival scaling and RSD were performed in two appointments with the help of hand curettes (Gracey Curettes, Hu-Friedy Instruments, Chicago, IL) and ultrasonic instruments to obtain smooth root surfaces. The gingivitis group underwent full mouth supragingival scaling up to $1 \mathrm{~mm}$ beyond the gingival margin with the help of ultrasonic instruments and corrections of plaque retentive margins. Participants were reinforced with $\mathrm{OHI}$ appropriate to their needs at recall visits. Approximately 9-12 weeks after the last periodontal treatment, both groups were reviewed and the questionnaire of OHIP-14 items was provided to all, which was self-completed. Appropriate periodontal treatment, including periodontal surgery, was prescribed for those individuals who still had sites with residual $\mathrm{PD} \geq 6 \mathrm{~mm}$ with bleeding on probing.

2.6. Statistical Analysis and Software Used. Only participants completing the questionnaires both at baseline and at the 9-12-week follow-up were included for statistical analysis. Collected data were entered into MS Excel 2007 and converted into the statistical software package SPSS 11.5 (SPSS, Chicago, IL, USA) for statistical analysis. Descriptive statistics like percentage, mean, SD, median, and IQR were calculated along with graphical and tabular presentation made. For inferential statistics, chi-squared test, Mann-Whitney $U$ test, and Wilcoxson's signed-rank test were applied to find out the significant difference between groups and within the group. The level of significance was considered ${ }^{*} p<0.05$ at $95 \%$ CI.

\section{Results}

This study comprised 49 adults with a mean age of $37 \pm 9.03$ (17-60 years) years. Their sociodemographic characteristics (SDC) are shown in Table 1.

The results concerning the impact of periodontal disease on OHRQoL are presented in Table 2. "Never" was reported in 10 out of 14 items of OHIP-14 by more than half of the patients before periodontal therapy. 13 out of 14 
TABLE 1: Association between groups and related SDC.

\begin{tabular}{|c|c|c|c|c|c|}
\hline \multirow{2}{*}{ Characteristics } & \multirow{2}{*}{ Category } & \multirow{2}{*}{ Total number (\%) } & \multicolumn{2}{|c|}{ Number (\%) } & \multirow{2}{*}{$p$ value } \\
\hline & & & Periodontitis & Gingivitis & \\
\hline \multirow{4}{*}{ Age group in years } & $>29$ & $9(100.0)$ & $0(0.0)$ & $9(100.0)$ & \multirow{4}{*}{ - } \\
\hline & $30-39$ & $18(100.0)$ & $5(27.8)$ & $13(72.2)$ & \\
\hline & $40-49$ & $17(100.0)$ & $14(82.4)$ & $3(17.6)$ & \\
\hline & $>50$ & $5(100.0)$ & $5(100.0)$ & $0(0.0)$ & \\
\hline Mean age in years \pm SD & & $49(100.0)$ & $43.46 \pm 7.6$ & $30.96 \pm 6.4$ & $0.001^{*}$ \\
\hline \multirow{2}{*}{ Gender } & Female & $23(46.9)$ & $12(52.2)$ & $11(47.8)$ & \multirow{2}{*}{0.674} \\
\hline & Male & $26(53.1)$ & $12(46.2)$ & $14(53.8)$ & \\
\hline \multirow{2}{*}{ Brushing frequency } & 1 & $33(67.3)$ & $18(54.5)$ & $15(45.5)$ & \multirow{2}{*}{0.263} \\
\hline & $\geq 2$ & $16(32.6)$ & $6(37.5)$ & $10(62.5)$ & \\
\hline \multirow{3}{*}{ Socioeconomic status } & Upper middle & $24(49.0)$ & $11(45.8 \%)$ & $13(54.2 \%)$ & \multirow{3}{*}{0.736} \\
\hline & Lower middle & $15(30.6)$ & $7(46.7 \%)$ & $8(53.3 \%)$ & \\
\hline & Upper lower & $10(20.4)$ & $6(60.0 \%)$ & $4(40.0 \%)$ & \\
\hline
\end{tabular}

${ }^{*}$ Statistically significant $p$ value $\left({ }^{*} p<0.05\right)$.

TABLE 2: Distribution of responses to OHIP-14 item scores for all participants.

\begin{tabular}{|c|c|c|c|c|c|c|c|c|c|c|}
\hline \multirow{2}{*}{ OHIP-14 item questions } & \multicolumn{5}{|c|}{ Before, $n(\%)$} & \multicolumn{5}{|c|}{ After, $n(\%)$} \\
\hline & 0 & 1 & 2 & 3 & 4 & 0 & 1 & 2 & 3 & 4 \\
\hline Trouble pronouncing words & $37(75.5)$ & $5(10.2)$ & $5(10.2)$ & $2(4.1)$ & 0.0 & $39(79.6)$ & $6(12.2)$ & $4(8.2)$ & 0.0 & 0.0 \\
\hline Taste has worsened & $42(85.7)$ & $5(10.2)$ & $2(4.1)$ & 0.0 & 0.0 & $46(93.9)$ & $2(4.1)$ & $1(2.0)$ & 0.0 & 0.0 \\
\hline Pain in mouth & $18(36.7)$ & $7(14.3)$ & $20(40.8)$ & $4(8.2)$ & 0.0 & $32(65.3)$ & $12(24.5)$ & $5(10.2)$ & 0.0 & 0.0 \\
\hline Uncomfortable eating food & $39(79.6)$ & $3(6.1)$ & $5(10.2)$ & $2(4.1)$ & 0.0 & $42(85.7)$ & $4(8.2)$ & $3(6.1)$ & 0.0 & 0.0 \\
\hline Self-consciousness & $17(34.7)$ & $11(22.4)$ & $11(22.4)$ & $9(18.4)$ & $1(2.0)$ & $28(57.1)$ & $12(24.5)$ & $5(10.2)$ & $3(6.1)$ & $1(2.0)$ \\
\hline Tense feeling & $14(28.6)$ & $10(20.4)$ & $14(28.6)$ & $7(14.3)$ & $4(8.2)$ & $20(40.8)$ & $14(28.6)$ & $10(20.4)$ & $4(8.2)$ & $1(2.0)$ \\
\hline Unsatisfactory diet & $47(95.9)$ & $1(2.0)$ & 0.0 & $1(2.0)$ & 0.0 & $48(98.0)$ & 0.0 & $1(2.0)$ & 0.0 & 0.0 \\
\hline Interruption of meals & $46(93.9)$ & $2(4.1)$ & 0.0 & $1(2.0)$ & 0.0 & $46(93.9)$ & $1(2.0)$ & $2(4.1)$ & 0.0 & 0.0 \\
\hline Difficulty relaxing & $32(65.3)$ & $9(18.4)$ & $4(8.2)$ & $3(6.1)$ & $1(2.0)$ & $39(79.6)$ & $7(14.3)$ & $2(4.1)$ & $1(2.0)$ & 0.0 \\
\hline Feeling embarrassed & $22(44.9)$ & $11(22.4)$ & $9(18.4)$ & $5(10.2)$ & $2(4.1)$ & $27(55.1)$ & $12(24.5)$ & $6(12.2)$ & $4(8.2)$ & 0.0 \\
\hline Irritable with others & $33(67.3)$ & $6(12.2)$ & $3(6.1)$ & $4(8.2)$ & $3(6.1)$ & $38(77.6)$ & $2(4.1)$ & $5(10.2)$ & $4(8.2)$ & 0.0 \\
\hline Difficulty doing usual jobs & $47(95.9)$ & $2(4.1)$ & 0.0 & 0.0 & 0.0 & $49(100.0)$ & 0.0 & 0.0 & 0.0 & 0.0 \\
\hline Less satisfaction & $47(95.9)$ & $1(2.0)$ & $1(2.0)$ & 0.0 & 0.0 & $47(95.9)$ & $1(2.0)$ & $1(2.0)$ & 0.0 & 0.0 \\
\hline Totally unable to function & $49(100.0)$ & 0.0 & 0.0 & 0.0 & 0.0 & $49(100.0)$ & 0.0 & 0.0 & 0.0 & 0.0 \\
\hline
\end{tabular}

0 : never; 1 : hardly ever; 2 : occasionally; 3 : fairly often; 4 : very often.

items of OHIP-14 reported "never" after NSPT. There were less reported answers "very often" from both groups. This indicated an impact of periodontal diseases on certain items of OHIP questionnaire and an improvement seen after the periodontal therapy in respective items and simultaneously in their QoL. The median (IQR) OHIP-14 total score at baseline for both groups was $7(\max .=22, \min .=0, \mathrm{IQR}$ =3-11). This decreased slowly following the completion of nonsurgical periodontal treatment, scoring a median of 3 ( maximum $=16$, minimum $=0, \mathrm{IQR}=1-7.5$ ). This indicated poor QoL of periodontal disease patients at baseline that improved significantly after periodontal treatment at 9-12 weeks ( $p$ value $<0.001$ ) (Table 3$)$. The overall median (IQR) reduction in percentage was found to be 46.6 (20.0-73.21) in periodontal disease patients. There was a significant difference between the groups at baseline $(p$ value $=0.015)$ but the difference was reduced after periodontal therapy
TABLE 3: Association of periodontal diseases OHIP-14 item score before and after NSPT" .

\begin{tabular}{lc}
\hline Test & Median score (IQR) \\
\hline Before & $7(3-11)(0-22)$ \\
After & $3(1-7.5)(0-16)$ \\
\hline
\end{tabular}

${ }^{\#}$ Wilcoxon's signed-rank test.

$(p$ value $=0.747)($ Table 4$)$. Both groups showed a statistically significant improvement in the score of OHIP-14 before and after treatment $(p$ value $=0.001)$ (Table 5$)$. The periodontitis group after scaling and RSD showed increased median (IQR) reduction in percentage, 51.9 (35.22-86.15), compared to median (IQR) percentage reduction in the gingivitis group after supragingival scaling, $27.2(0.00-50.00)$. The reduction in percentage was significant between the groups. 
TABLE 4: Association between the groups of OHIP-14 item score before and after NSPT ${ }^{\dagger}$.

\begin{tabular}{lccc}
\hline Test & \multicolumn{2}{c}{ Median (IQR) } & \\
& $\begin{array}{c}\text { Periodontitis } \\
\text { (scaling and RSD) }\end{array}$ & $\begin{array}{c}\text { Gingivitis } \\
\text { (supragingival scaling) }\end{array}$ & $p$ value \\
\hline Before & $8.50(4.25-15.00)$ & $4.00(2.50-10.00)$ & $0.015^{*}$ \\
After & $3.00(1.00-7.50)$ & $2.00(1.00-7.50)$ & 0.747 \\
\hline
\end{tabular}

${ }^{\dagger}$ Mann-Whitney $U$ test. ${ }^{*}$ Statistically significant $p$ value $\left({ }^{*} p<0.05\right)$.

TABLE 5: Association between intragroups of OHIP-14 item score before and after NSPT ${ }^{\ddagger}$.

\begin{tabular}{lccc}
\hline \multirow{2}{*}{ Group } & \multicolumn{2}{c}{ Median (IQR) } & Before \\
& After & \\
\hline Periodontitis & $8.50(4.25-15.00)$ & $3.00(1.00-7.50)$ & $<0.001^{*}$ \\
Gingivitis & $4.00(2.5-10.00)$ & $2.00(1.00-7.50)$ & $0.001^{*}$ \\
\hline${ }^{\ddagger}$ Wilcoxson's signed-rank test. ${ }^{*}$ Statistically significant $p$ value $\left.{ }^{*} p<0.05\right)$
\end{tabular}

\section{Discussion}

This study comprised 49 adults with a mean age of $37.0 \pm 9.03$ years. This study showed a significant impact of periodontal diseases on OHRQoL. The result of this study is in agreement with findings of most of the other studies $[2,6,27-30]$ showing poorer QoL in periodontal disease individuals. This study compared the effect on change in OHRQoL using OHIP-14 score before and after NSPT. The results were statistically significant to show enhanced improvement after receiving the therapy in periodontal disease individuals. The findings were similar to other studies showing notable improvement $[6,16,22,27,31]$. Median (IQR) OHIP-14 scores in this study reduced from $7(3-11)$ at baseline to 3 (1-7.5) 9-12 weeks after treatment ( $p$ value $<0.001$ ). These scores of median reduction are comparable with the findings reported in different populations of periodontal disease patients worldwide $[6,18,31]$.

The assessment of NSPT is generally made in no less than 1 to 3 months; therefore, in this study, OHRQoL was analyzed after 9-12 weeks [32]. No adjunctive therapies to scaling and RSD were compared in this study. A recent meta-analysis has shown that scaling and root planning result in $0.5 \mathrm{~mm}$ improvement in clinical attachment level (CAL) at a moderate level of certainty against 0.2 to $0.6 \mathrm{~mm}$ improvement in CAL in combination with adjuncts [33]. A Nepali version of OHIP-14 item score was used. The OHIP was designed to provide a comprehensive measure of oral functional limitations, oral pain, and discomfort and the psychological and behavioral impacts of oral conditions [24], and currently with the exploratory factor analysis of Oral Health Impact Profile there is evidence for a more differentiated four-dimensional structure of OHIP items. It is one of the most widely used instruments and has been used to assess the impact of periodontal disease as well [5, $6,18]$. The OHIP-14 item scores were significantly associated with periodontal symptoms which include swollen, sore, or receding gums, toothache, loose teeth, and bad breath [5].
In this study, the impact of chronic periodontal diseases and their particular treatment were compared before and after NSPT. The periodontitis group had higher mean age of $43.46 \pm 7.6$ years compared to the gingivitis group. All other sociodemographic data were similar with participants belonging to the upper middle socioeconomic class. Healthy controls were not included in this study as it was expected that they would have better OHRQoL than diseased individuals. A study done by Jowett et al. [6] in 2009 compared between two cohorts with periodontal disease but it was done only for 7 days and nonsurgical phases of treatment were not compared. In this study, there was a significant difference between the groups at baseline $(p=0.015)$. This can be explained by the fact that, in the periodontitis group, the disease symptoms were more severe regarding pain, mobility, unesthetic loss of papilla, greater probing depths, and bleeding gums as compared to the gingivitis group that reported very few of these impacts. Both groups showed improvement after periodontal therapy on OHRQoL; however, the treatment done by scaling and RSD showed increased median (IQR) reduction in percentage, 51.92 (35.22-86.15), compared with the gingivitis group after supragingival scaling, $27.27(0.00-50.00)$. Studies that compare both of these treatments separately are scarce, but recently a study done by Mendez et al. in 2016 [22] showed improvement after supragingival treatment, but they did not describe RSD as a separate phase of treatment. The periodontitis group had higher median (IQR) OHIP14 score signifying poor QoL. The findings of this study are comparable to studies showing that extent/severity affects QoL $[5,12,34]$ and greater improvement is seen in patients with greater severity $[2,6,18,35]$.

Researchers consider the change in oral health to be assessed by one score as meaningful, but periodontal diseases are complex diseases and there is a danger in the interpretation of the results as one because one aspect may have improved and another might have deteriorated [36]. Pain in the mouth was not reported "very often" by both groups, but $40.8 \%$ of the patients reported "occasional pain" initially; after treatment, only $10.2 \%$ of the patients reported pain. This signified an improvement perceived by patients and is in agreement with most of the studies showing improvement after periodontal therapy $[18,31]$. Significant improvement in pain was also observed in OHRQoL measures other than OHIP-14 [37-39]. Although a reduction in the frequency of problems of orofacial pain and improvement in clinical signs of periodontal pathology can be expected after NSPT, there are other inevitable outcomes of periodontal therapy such as cervical sensitivity, gingival recession, pathologically migrated teeth, and loss of papillae which may affect a patient's appearance as well as having an impact psychosocially. In the periodontitis group, $37.4 \%$ of the patients were irritable with others that ranged from a response of "hardly ever to very often" that reduced to $24.9 \%$ after treatment and $58.3 \%$ of patients were embarrassed in front of other people that improved to $41.7 \%$ after treatment. Marked differences were not observed in these aspects in periodontitis patients as sometimes NSPT might not be enough for treatment but a tendency to improvement was seen. This signified that chronic periodontitis may interfere with psychosocial 
aspects of periodontal disease patients and therefore we may challenge the perception of chronic periodontitis as a silent disease [40]. However, in the gingivitis group, patients had less severe symptoms and complained mostly of enlarged gums, malodor, and bleeding. These symptoms were reduced after treatment and improvements were appreciable by the patients. Also, in the periodontitis group, $79.2 \%$ of the patients were conscious of their appearance, with $75.1 \%$ feeling tensed, which improved to $45.8 \%$ and $58.4 \%$ after treatment, respectively. In the gingivitis group, $52.0 \%$ of the patients were conscious, with $68.0 \%$ feeling tensed, which improved to $36.0 \%$ and $44.0 \%$, respectively. It may be therefore predicted that orofacial appearance has a definite impact on quality of life of periodontal disease patients and frequent visits and/or intervention by the therapist might have provided them with some form of gain in confidence and positive feeling. The results are comparable to other studies showing similar results $[18,31]$.

The oral function was not affected to a great extent as most of the patients reported "never" for difficulty in speech $(75.5 \%)$ and altered taste $(85.7 \%)$ in both groups. Findings are comparable to studies demonstrating the least improvement in this dimension $[18,31]$. The reason might be that patients were dentulous and only a few patients reported discomfort with mobility. Aggressive periodontitis patients that can be expected to have poorer QoL and affected functionality were also not included $[5,41]$. Changes in different dimensions may be affected by the signs and symptoms reported but clearly both treatment protocols provided improved periodontal health and helped to bring about a change in an individual's QoL. The participants in this study were of a younger age group. This may prove that even younger individual's QoL is affected, which might improve after therapy.

One of the major limitations of this study is the high prevalence of subjects with zero scores which may compromise the ability of OHIP-14 to detect within-subject changes. Nevertheless, Nepalese language validation of other measures such as condition specific OHQoL tool, GOHAI (General Oral Health Assessment Index), and OHQoL-UK (Oral Health Quality of Life-UK) which reports positive effect of oral conditions and not just frequency is highly desirable. Further long-term studies with larger populations are required to address the above limitations.

In conclusion, this study clearly indicated that periodontal diseases negatively impact an individual's quality of life in many aspects, especially of those patients suffering from chronic generalized periodontitis compared to patients with gingivitis. Nonsurgical periodontal therapy has a constructive role to play to ameliorate the impact, with significant changes observed for full mouth scaling and root surface debridement procedure.

\section{Additional Points}

The clinical efficacy after periodontal treatment is irrefutable, but along with the treatment provided, if the clinicians can help understand the patient's perspective in a better way, it can definitely provide a holistic approach towards patient's oral care.

\section{Conflicts of Interest}

The authors report no conflicts of interest regarding the publication of this paper.

\section{References}

[1] M. C. Downer, "The changing pattern of dental disease over 50 years," British Dental Journal, vol. 185, no. 1, pp. 36-41, 1998.

[2] S. L. Buset, C. Walter, A. Friedmann, R. Weiger, W. S. Borgnakke, and N. U. Zitzmann, "Are periodontal diseases really silent? A systematic review of their effect on quality of life," Journal of Clinical Periodontology, vol. 43, no. 4, pp. 333344, 2016.

[3] W. M. Thomson, A. Sheiham, and A. J. Spencer, "Sociobehavioral aspects of periodontal disease," Periodontology 2000, vol. 60, no. 1, pp. 54-63, 2012.

[4] US Department of Health and Human Services, Oral Health in America: A Report of the Surgeon General-Executive Summary, US Department of Health and Human Services, National Institute of Dental and Craniofacial Research, National Institutes of Health, Rockville, Md, USA, 2000.

[5] S. K. S. Ng and W. K. Leung, "Oral health-related quality of life and periodontal status," Community Dentistry and Oral Epidemiology, vol. 34, no. 2, pp. 114-122, 2006.

[6] A. K. Jowett, M. T. S. Orr, A. Rawlinson, and P. G. Robinson, "Psychosocial impact of periodontal disease and its treatment with 24-h root surface debridement," Journal of Clinical Periodontology, vol. 36, no. 5, pp. 413-418, 2009.

[7] K. A. Atchison and T. A. Dolan, "Development of the geriatric oral health assessment index," Journal of Dental Education, vol. 54, no. 11, pp. 680-687, 1990.

[8] C. McGrath and R. Bedi, "An evaluation of a new measure of oral health related quality of life-OHQoL-UK(W)," Community Dental Health, vol. 18, no. 3, pp. 138-143, 2001.

[9] S. Adulyanon, J. Vourapukjaru, and A. Sheiham, "Oral impacts affecting daily performance in a low dental disease Thai population," Community Dentistry and Oral Epidemiology, vol. 24, no. 6, pp. 385-389, 1996.

[10] G. D. Slade, "Derivation and validation of a short-form oral health impact profile," Community Dentistry and Oral Epidemiology, vol. 25, no. 4, pp. 284-290, 1997.

[11] P. F. Allen, A. S. McMillan, and D. Locker, "An assessment of sensitivity to change of the Oral Health Impact Profile in a clinical trial," Community Dentistry and Oral Epidemiology, vol. 29, no. 3, pp. 175-182, 2001.

[12] I. Needleman, C. McGrath, P. Floyd, and A. Biddle, "Impact of oral health on the life quality of periodontal patients," Journal of Clinical Periodontology, vol. 31, no. 6, pp. 454-457, 2004.

[13] D. Locker, D. Matear, M. Stephens, H. Lawrence, and B. Fayne, "Comparison of the GOHAI and OHIP-14 as measures of the oral health-related quality of life of the elderly," Community Dentistry and Oral Epidemiology, vol. 29, no. 5, pp. 373-381, 2001.

[14] M. Vikram and V. P. Singh, "Translation and validation of the Nepalese version of oral health impact profile (OHIP-14) questionnaire," Oral Biology and Dentistry, vol. 2, no. 3, 2014.

[15] J. A. Jones, N. R. Kressin, D. R. Miller, M. B. Orner, R. I. Garcia, and A. Spiro III, "Comparison of patient-based oral health outcome measures," Quality of Life Research, vol. 13, no. 5, pp. 975-985, 2004. 
[16] O. Ozcelik, M. C. Haytac, and G. Seydaoglu, "Immediate postoperative effects of different periodontal treatment modalities on oral health-related quality of life: a randomized clinical trial," Journal of Clinical Periodontology, vol. 34, no. 9, pp. 788-796, 2007.

[17] A. Saito, K. Ota, Y. Hosaka et al., "Potential impact of surgical periodontal therapy on oral health-related quality of life in patients with periodontitis: a pilot study," Journal of Clinical Periodontology, vol. 38, no. 12, pp. 1115-1121, 2011.

[18] F. Brauchle, M. Noack, and E. Reich, "Impact of periodontal disease and periodontal therapy on oral health-related quality of life," International Dental Journal, vol. 63, no. 6, pp. 306-311, 2013.

[19] K. Öhrn and B. Jönsson, "A comparison of two questionnaires measuring oral health-related quality of life before and after dental hygiene treatment in patients with periodontal disease," International Journal of Dental Hygiene, vol. 10, no. 1, pp. 9-14, 2012.

[20] A. Bajwa, T. L. Watts, and J. T. Newton, "Health control beliefs and quality of life considerations before and during periodontal treatment," Oral Health \& Preventive Dentistry, vol. 5, no. 2, pp. 101-104, 2007.

[21] G. Tsakos, E. Bernabé, F. D’Aiuto et al., “Assessing the minimally important difference in the oral impact on daily performances index in patients treated for periodontitis," Journal of Clinical Periodontology, vol. 37, no. 10, pp. 903-909, 2010.

[22] M. Mendez, P. Melchiors Angst, A. Stadler, R. Oppermann, and S. Gomes, "Impacts of supragingival and subgingival periodontal treatments on oral health-related quality of life," International Journal of Dental Hygiene, 2016.

[23] A. G. Ghosh and G. Tusharkanti, "Modification of Kuppuswamy's socioeconomic status scale in context to Nepal," Indian Pediatrics, vol. 46, no. 12, pp. 1104-1105, 2009.

[24] G. D. Slade and A. J. Spencer, "Development and evaluation of the Oral Health Impact Profile," Community Dental Health, vol. 11, no. 1, pp. 3-11, 1994.

[25] D. Locker, A. Jokovic, and M. Clarke, "Assessing the responsiveness of measures of oral health-related quality of life," Community Dentistry and Oral Epidemiology, vol. 32, no. 1, pp. 10-18, 2004.

[26] M. T. John, D. R. Reissmann, L. Feuerstahler et al., "Exploratory factor analysis of the oral health impact profile," Journal of Oral Rehabilitation, vol. 41, no. 9, pp. 635-643, 2014.

[27] S. Shanbhag, M. Dahiya, and R. Croucher, "The impact of periodontal therapy on oral health-related quality of life in adults: a systematic review," Journal of Clinical Periodontology, vol. 39, no. 8, pp. 725-735, 2012.

[28] R. Al Habashneh, Y. S. Khader, and S. Salameh, "Use of the Arabic version of Oral Health Impact Profile-14 to evaluate the impact of periodontal disease on oral health-related quality of life among Jordanian adults," Journal of oral science, vol. 54, no. 1, pp. 113-120, 2012.

[29] J. Durham, H. M. Fraser, G. I. McCracken, K. M. Stone, M. T. John, and P. M. Preshaw, "Impact of periodontitis on oral health-related quality of life," Journal of Dentistry, vol. 41, no. 4, pp. 370-376, 2013.

[30] B. Jönsson and K. Öhrn, "Evaluation of the effect of nonsurgical periodontal treatment on oral health-related quality of life: estimation of minimal important differences 1 year after treatment," Journal of Clinical Periodontology, vol. 41, no. 3, pp. 275-282, 2014.
[31] R. M. S. Wong, S. K. S. Ng, E. F. Corbet, and W. Keung Leung, "Non-surgical periodontal therapy improves oral health-related quality of life," Journal of Clinical Periodontology, vol. 39, no. 1, pp. 53-61, 2012.

[32] A. Badersten, R. Nilveus, and J. Egelberg, "Effect of nonsurgical periodontal therapy II. Severely advanced periodontitis," Journal of Clinical Periodontology, vol. 11, no. 1, pp. 63-76, 1984.

[33] C. J. Smiley, S. L. Tracy, E. Abt et al., "Systematic review and meta-analysis on the nonsurgical treatment of chronic periodontitis by means of scaling and root planing with or without adjuncts," Journal of the American Dental Association, vol. 146, no. 7, pp. 508-524, 2015.

[34] E. Bernabé and W. Marcenes, "Periodontal disease and quality of life in British adults," Journal of Clinical Periodontology, vol. 37, no. 11, pp. 968-972, 2010.

[35] T. Drumond-Santana, F. O. Costa, E. G. Zenóbio, R. V. Soares, and T. D. Santana, "Impact of periodontal disease on quality of life for dentate diabetics," Cadernos de Saude Publica, vol. 23, no. 3, pp. 637-644, 2007.

[36] C. R. B. Joyce, M. M. Hannah, and A. O. Ciaran, Individual Quality of Life: Approaches to Conceptualization and Assessment, Harwood Academic Publishers, 1999.

[37] A. Saito, Y. Hosaka, M. Kikuchi et al., "Effect of initial periodontal therapy on oral health-related quality of life in patients with periodontitis in Japan," Journal of Periodontology, vol. 81, no. 7, pp. 1001-1009, 2010.

[38] A. Makino-Oi, Y. Ishii, T. Hoshino et al., "Effect of periodontal surgery on oral health-related quality of life in patients who have completed initial periodontal therapy," Journal of Periodontal Research, vol. 51, no. 2, pp. 212-220, 2016.

[39] M. Åslund, J. Suvan, D. R. Moles, F. D’Aiuto, and M. S. Tonetti, "Effects of two different methods of non-surgical periodontal therapy on patient perception of pain and quality of life: a randomized controlled clinical trial," Journal of Periodontology, vol. 79, no. 6, pp. 1031-1040, 2008.

[40] J. Cunha-Cruz, P. P. Hujoel, and N. R. Kressin, "Oral healthrelated quality of life of periodontal patients," Journal of Periodontal Research, vol. 42, no. 2, pp. 169-176, 2007.

[41] A. C. Araujo, E. S. Gusmao, J. E. Batista, and R. Cimoes, "Impact of periodontal disease on quality of life," Quintessence International, vol. 41, no. 6, pp. el11-e118, 2010. 


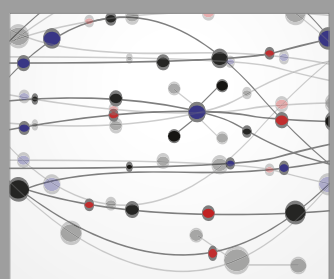

The Scientific World Journal
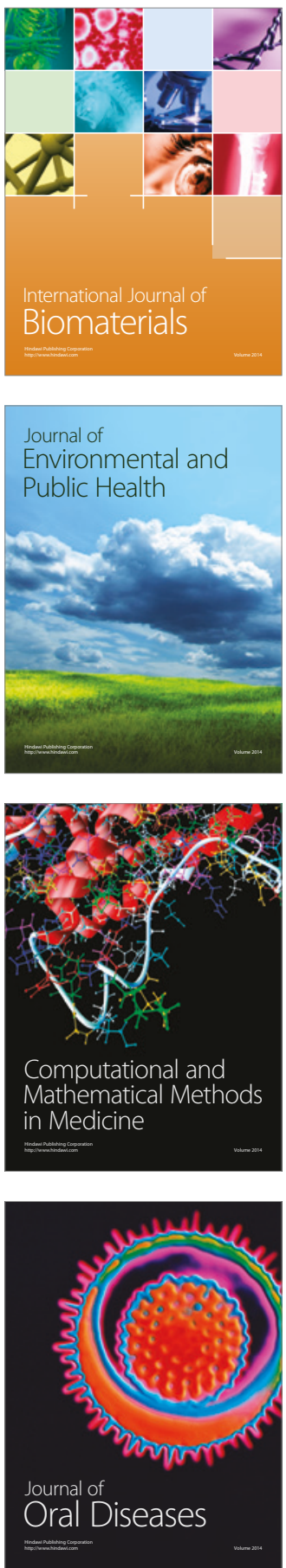
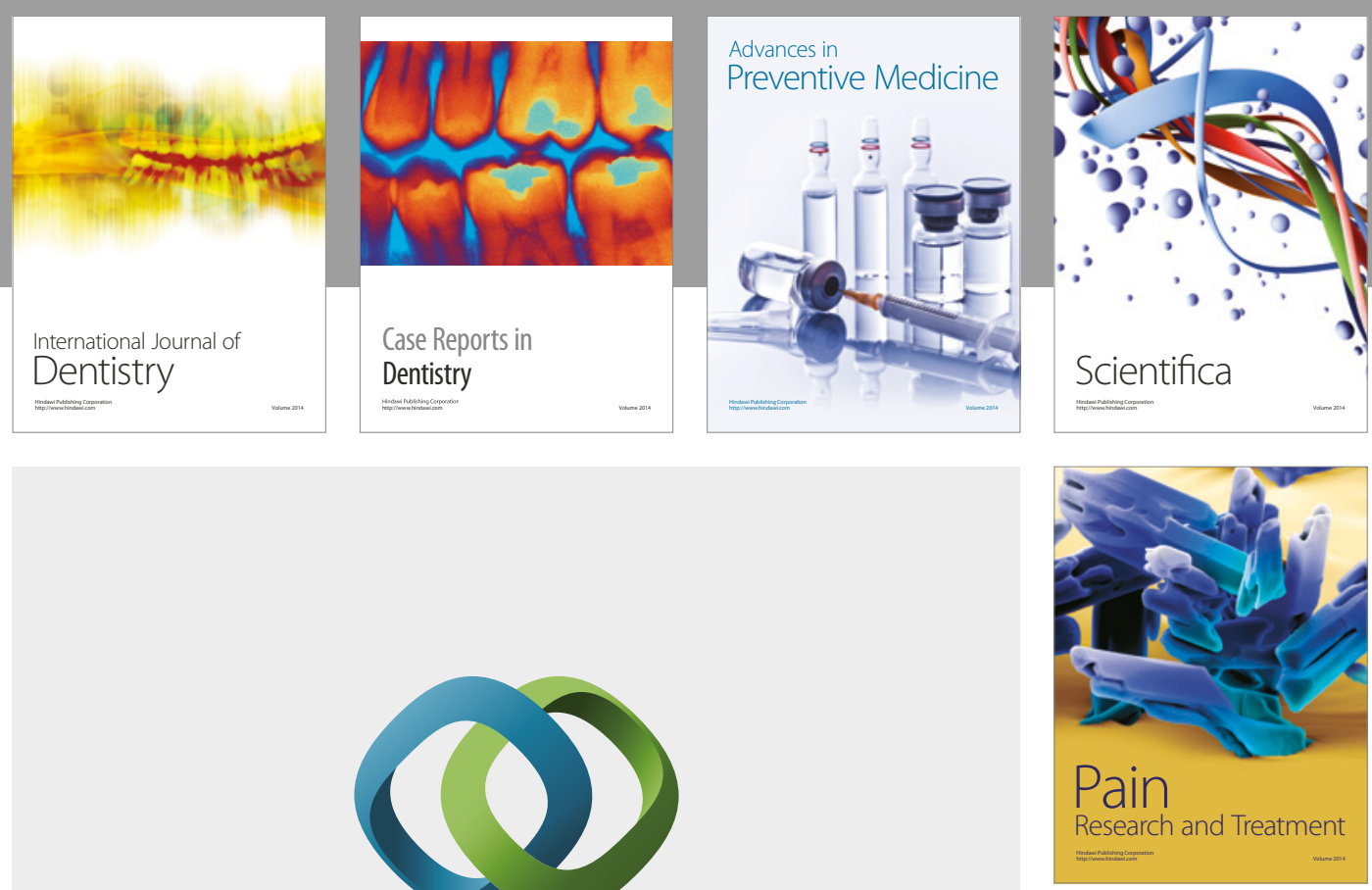

\section{Hindawi}

Submit your manuscripts at

https://www.hindawi.com
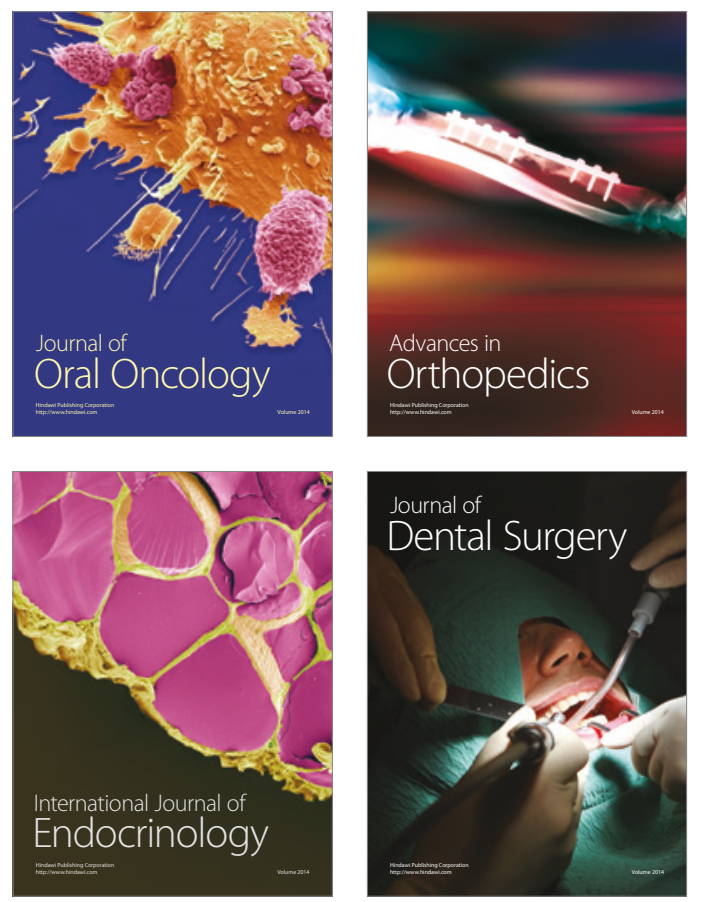
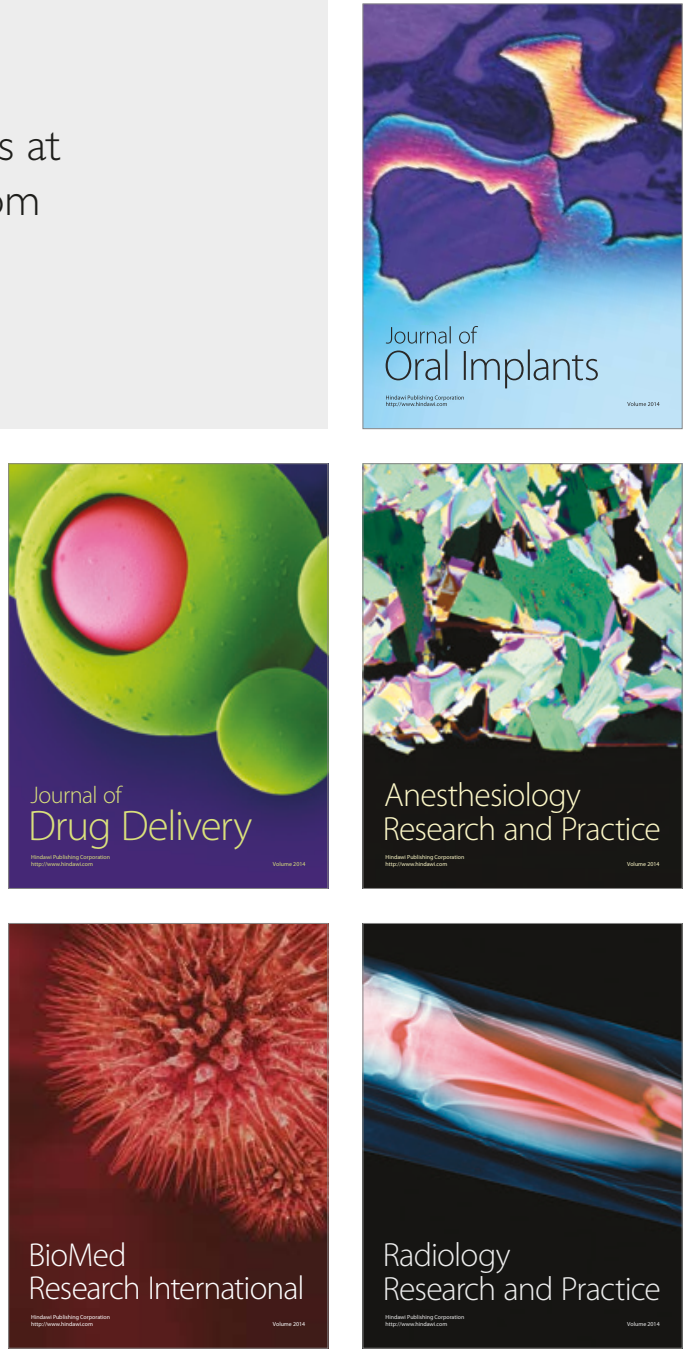